\title{
Additive Effect of Intravascular Complement Activation and Brief Episodes of Hypoxia in Producing Increased Permeability in the Rabbit Lung
}

Gary L. Larsen, Robert O. Webster, G. Scott Worthen, R. Steven Gumbay, and Peter M. Henson

Departments of Pediatrics and Medicine, National Jewish Hospital/National Asthma Center, and School of Medicine,

University of Colorado Health Sciences Center, Denver, Colorado 80206

\begin{abstract}
Systemic complement activation with intravascularly administered cobra venom factor (CVF) or infusion of either zymosanactivated rabbit plasma or a fifth component of complement fragment with anaphylatoxin activity in the rabbit have not caused significant increases in bronchoalveolar lavage albumin in rabbits (Webster, R. O., G. L. Larsen, B. C. Mitchell, A. J. Goins, and P. M. Henson. 1982. Am. Rev. Respir. Dis. 125:335-340). To assess if another stimulus (hypoxia) acting in concert with complement activation can produce significant lung injury, rabbits were challenged with CVF alone, $10 \mathrm{~min}$ of $12 \%$ oxygen alone, or CVF followed by a 10-min exposure to $12 \%$ oxygen. Either stimulus alone caused no significant changes in arterial oxygen, pulmonary resistance, or dynamic compliance during the $240 \mathrm{~min}$ of observation after either stimulus, and neither stimulus alone caused increased albumin accumulation in bronchoalveolar lavage over a 30 -min period at the end of the experiment. However, the combination of insults significantly altered arterial oxygen, pulmonary resistance, and dynamic compliance while also increasing albumin and neutrophils recovered by lavage. The increase in lavage albumin did not appear to be due to hemodynamic events in that the pulmonary artery pressure increased acutely after CVF infusion and again during the hypoxic exposure, but was normal when albumin accumulation in the lung was measured. Neutrophil depletion with nitrogen mustard abolished all of these changes induced by CVF plus hypoxia. In addition, meclofenamate pretreatment and infusion during the 4-h study abolished the increases in lavage albumin and neutrophils as well as the increase in pulmonary artery pressure after CVF. Meclofenamate pretreatment did not, however, block accumulation of albumin in the lung (interstitium). We conclude that complement activation, as an isolated event, will not cause a significant increase in lavage albumin in this model. However, combining complement activation with an episode of hypoxia will lead to an increase in lavage albumin that is dependent on the presence of neutrophils for its expression. Meclofenamate treatment will prevent increases in lavage albumin and neutrophils while not preventing albumin accumulation in the lung
\end{abstract}

This work was performed by Dr. Worthen during the tenure of a Clinician-Scientist Award from the American Heart Association, with funds provided in part by the Colorado Heart Association. Address reprint requests to Dr. Larsen, Department of Pediatrics. Dr. Webster's current address is Pulmonary Division, St. Louis University School of Medicine, St. Louis, MO 63104.

Received for publication 22 May 1984 and in revised form 2 October 1984.

J. Clin. Invest.

(c) The American Society for Clinical Investigation, Inc.

0021-9738/85/03/902/09 \$1.00

Volume 75, March 1985, 902-910 (interstitium), suggesting a product of the cyclooxygenase pathway of arachidonic acid metabolism is needed to produce movement of albumin and/or neutrophils across the alveolar epithelium in this model.

\section{Introduction}

Some recent observations have led to the hypothesis that intravascular complement activation can lead to neutrophil sequestration in pulmonary vessels with subsequent lung injury. Craddock and co-workers (1) found uremic patients undergoing hemodialysis developed neutropenia and lung dysfunction. In sheep and rabbits, they demonstrated that plasma incubated with dialyzer cellophane produced neutropenia and hypoxemia when infused into the test animals, with pulmonary histology showing gross intravascular leukostasis. Analysis of the plasma demonstrated complement activation had occurred. Hammerschmidt and co-workers (2) reported that, in patients at risk for developing the adult respiratory distress syndrome, neutrophil-aggregating activity in the plasma correlated significantly with the development of this syndrome.

Complement fragments derived from the fifth component of complement, $(\mathrm{C} 5)^{1}$ have been implicated as being important in causing these observed changes in lung structure and function for several reasons. First, the phlogistic potential of both the $\mathrm{C} 5$ fragment with anaphylatoxin activity (C5a) and the $\mathrm{C} 5$ fragment formed by removal of the $\mathrm{C}$-terminal arginine from $\mathrm{C} 5 \mathrm{a}$, in terms of producing neutrophil chemotaxis, enzyme release, and oxygen radical production, are well described $(3,4)$. In addition, Sacks and co-workers (5) suggested that C5a-stimulated granulocytes can cause endothelial cell damage in vitro. Complement activation in an intrapulmonary but extravascular location has been noted by us (6) and others (7-9) to produce pulmonary inflammation. Thus, although the inflammatory potential of C5 fragments in vitro and in an intrapulmonary location is well-established, their role in producing significant lung injury in an intravascular location is more open to debate. Although Till and associates (10) have found complement activation with cobra venom factor (CVF) in rats leads to lung injury, we reported that, in a rabbit model, the infusion of rabbit $\mathrm{C} 5 \mathrm{a}$, zymosan-activated rabbit plasma, or CVF were insufficient stimuli to produce alterations in lavage protein (albumin) despite causing pulmonary leukocyte sequestration and migration of neutrophils into the airspaces of the lung (11).

The present study was undertaken to determine whether

1. Abbreviations used in this paper: $C_{\mathrm{dyn}}$, dynamic compliance; CVF, cobra venom factor; C5, fifth component of complement; C5a, C5 fragment with anaphylatoxin activity; $\mathrm{PaCO}_{2}$, partial pressure of carbon dioxide in arterial blood; $\mathrm{PaO}_{2}$, partial pressure of oxygen in arterial blood; PAP, pulmonary artery pressure; RBC, erythrocyte; $R_{\mathrm{L}}$, pulmonary resistance; RSA, rabbit serum albumin. 
another stimulus in addition to complement activation could lead to significant lung injury. Wedmore and Williams (12) described increased vascular permeability in rabbit skin when chemotactic fragments were injected with prostaglandin $E_{2}$, and we noted increased vascular permeability in the lung when complement activation with CVF was followed by infusion of prostaglandin $\mathrm{E}_{2}$ (13). The possible additive effect of chemotactic fragments and vasodilator prostaglandins in causing increased injury in both the skin and lung, plus the observation of Said and co-workers (14) that hypoxia leads to prostaglandin synthesis, led to the current study where hypoxia was selected as the stimulus to combine with complement activation in an attempt to produce lung injury.

\section{Methods}

Animals. New Zealand white rabbits weighing $2.5-3.4 \mathrm{~kg}$ were used in the experiments.

Experimental design. Rabbits were anesthetized with sodium methohexital (Eli Lilly \& Co., Indianapolis, IN) and intubated with a cuffed endotracheal tube, which was secured into position with adhesive tape. An esophogeal balloon was inserted for use in determination of pulmonary functions (see below). Once anesthetized, an indwelling catheter was placed in the femoral artery. Sterile, pyrogen-free saline was infused to maintain an open catheter as well as the rabbit's circulatory system in an isovolume state. Blood samples were serially removed via a three-way stopcock for blood gas determinations, leukocyte counts, and complement assays. In selected experiments, respiratory function was not assessed, but hemodynamic events were monitored (see below). For all experiments, body temperature was monitored rectally, and maintained at $39.4 \pm 0.2^{\circ} \mathrm{C}$ by a heating pad placed under the rabbit.

After surgery, the endotracheal tube was attached to a size 00 pneumotach (Hewlett-Packard model 21069B, Palo Alto, CA), which was attached to a Harvard respirator (Harvard Apparatus Co., Inc. S. Natick, MA). The animals breathed either room air or $12 \%$ oxygen delivered by the small animal respirator. Anesthesia was maintained by intermittent slow infusions of sodium methohexital. After obtaining stable blood gas and pulmonary function baselines, or stable hemodynamic measurements when these variables were monitored, the rabbits were injected with the test stimulus via the marginal ear vein as a bolus. In all experiments, CVF $(300 \mathrm{U})$ or saline was injected at the beginning of the experiments while the rabbits were ventilated with room air. If they were subjected to the 10 -min exposure to $12 \%$ oxygen, this was done $10 \mathrm{~min}$ after the bolus of intravenous saline (control) or CVF. They breathed the hypoxic mixture through the ventilator by connecting a weather balloon filled with the hypoxic mixture to the intake portal of the ventilator. After the 10-min hypoxia exposure, they were ventilated with room air for the remainder of the experiment. Assessment of lung injury was through evaluation of lung function (blood gases, lung mechanics), analysis of bronchoalveolar lavage for cells, and radiolabeled albumin, and assessment of lung structure, as detailed below.

Measurements of pulmonary functions in rabbits. Arterial blood gases (the partial pressure of arterial oxygen $\left[\mathrm{PaO}_{2}\right]$, the partial pressure of arterial carbon dioxide $\left[\mathrm{PaCO}_{2}\right]$, and the hydrogen ion concentration [pH]) were measured on an ABL2 Acid Base Laboratory (Radiometer, Copenhagen) using heparinized blood, with correction for the body temperature of the rabbit.

As noted above, the intubated animal breathed room air or $12 \%$ oxygen delivered by the respirator through a pneumotach. 5-10 min before each measurement of lung function, three forced inflations of the lungs to two times the tidal volume were performed to establish a lung volume history (15). Transpulmonary pressure was recorded employing a Hewlett-Packard 270 differential pressure transducer by simultaneously measuring esophageal pressure with an esophageal balloon, and the pressure at the proximal end of the endotracheal tube through a T-piece. As shown by Caldwell and Fry (16), esophageal pressure in the rabbit closely approximates pleural pressure. The flow signal was integrated to volume, and from simultaneous measurements of inspiratory and expiratory flow, transpulmonary pressure, and tidal volume, a Hewlett-Packard 8816A respiratory analyzer computed dynamic compliance $\left(C_{\mathrm{dyn}}\right)$ by the method of von Neergard and Wirz (17), and pulmonary resistance $\left(R_{\mathrm{L}}\right)$ as outlined by Mead and Whittenberger (18). Validation of the automated determination of $R_{\mathrm{L}}$ by electrical subtraction and computation of $C_{\mathrm{dyn}}$ by dividing volume by transpulmonary pressure at zero flow, as employed by this equipment, was recently published by Drazen and co-workers (19).

Measurement of hemodynamic functions in rabbits. For experiments where hemodynamic events were monitored, a PE-90 catheter (Clay Adams, Parsippany, NJ) shaped as described by Owen-Thomas and Reeves (20) was inserted through the right jugular vein into the right ventricle, and positioned in the pulmonary artery. Characteristic pressure tracings were used to determine position, confirmed in a few animals by fluoroscopy and radio-opaque dye infusion. The catheter was connected to a Statham P23b pressure transducer (Gould, Inc., Oxnard, CA) to permit pressure monitoring. A No. 4 French thermister-tipped end-hole catheter specially constructed by American Catheter (through Electronics for Medicine, Honeywell Inc., White Plains, NY) was inserted through a femoral artery to $20 \mathrm{~cm}$, and connected to a Statham P23b transducer. The thermister leads were connected to a cardiac output computer (Electronics for Medicine, Honeywell Inc., model DTCCO-07) modified by the manufacturer to permit measurement of cardiac output in small animals. Pressure transducers and thermister output from the cardiac output computer were recorded on an Electronics for Medicine VR-6 recorder (Electronics for Medicine, Honeywell Inc.). Cardiac outputs were determined by injecting $1.0 \mathrm{ml}$ of room-temperature saline into the pulmonary artery catheter. Temperature of the injectate was determined by a probe from the cardiac output computer. Cardiac outputs determined by this method were reproducible and constant over several hours if core body temperature was maintained $\pm 0.2^{\circ} \mathrm{C}$. To ensure that this method resulted in accurate outputs, results using the above techniques were compared with cardiac outputs determined by the Fick method (21). To accomplish this, a one-way valve with $10 \mathrm{ml}$ of dead space was constructed so the rabbit could breathe room air and exhale into a collection bag. During a 2-min collection, simultaneous arterial and mixed venous blood samples were taken for measurement of the arteriovenous content difference for oxygen. Thermodilution cardiac output was measured during this period. Exhaled gas was measured for oxygen content by using a mass spectrometer (model 1100 Medical Gas Analyzer, PerkinElmer, Pomona, CA) and for volume. From these data, Fick and thermodilution cardiac outputs were determined. An excellent correlation was noted between the two (Worthen, G. S., unpublished observations). During experiments, pressure was measured continually and recorded at 5-10-min intervals while cardiac output was measured every $10-30 \mathrm{~min}$.

Permeability changes in rabbit lungs. In initial experiments, permeability was assessed as previously outlined (11). 30 min before the rabbits were killed, they were injected intravenously with $10 \mu \mathrm{g}$ of ${ }^{125} \mathrm{I}$ rabbit serum albumin (RSA), $0.3 \mu \mathrm{Ci} / \mu \mathrm{g}$ of protein in $1 \mathrm{ml}$ of normal saline. At death, samples of blood and lavage fluids were obtained, and radioactivity was measured in a gamma scintillation counter (Beckman Instruments, Inc., Fullerton, CA). The data was expressed as the radioactivity (counts per minute) in $1 \mathrm{ml}$ of lavage fluid divided by either the injected counts (counts per minute per milliliter $/ 10^{6}$ counts per minute of injected) or the radioactivity in $1 \mathrm{ml}$ of plasma (lavage/plasma ratio of ${ }^{125}$ I-RSA in counts per minute per milliliter of lavage/counts per minute per milliliter of plasma).

In subsequent experiments, a more detailed determination of permeability changes within the lung was employed using a modification of a gravimetric determination described by Pearce and associates (22). For this method, ${ }^{59} \mathrm{Fe}$-labeled erythrocytes (RBC) were used as a marker of intravascular blood volume instead of hemoglobin as employed by Pearce et al. (22). The details of in vivo labeling of RBC 
with this radiolabel are given below. In these experiments, ${ }^{125}$ I-RSA was injected as detailed above, i.e., $30 \mathrm{~min}$ before the death of the animal. In addition, the ${ }^{59} \mathrm{Fe}-\mathrm{RBC}$ were injected $15 \mathrm{~min}$ before death. Samples obtained at the end of the experiment included blood, lung (right upper lobe), and lavage for determination of radioactivity $\left({ }^{125} \mathrm{I}\right.$ RSA and ${ }^{59} \mathrm{Fe}-\mathrm{RBC}$ ) corrected for crossover. In addition, wet and dry weight of both lung and blood were determined. Values for lung weights (wet or dry) and lung albumin were computed by subtracting the contribution of lung blood to these values.

Serum albumin: preparation and radiolabeling. Rabbit albumin was isolated following TEAE-cellulose chromatography of rabbit C5 (23). It was purified further by molecular sieving on Sephadex G-100. Protein purity was determined by SDS polyacrylamide gel electrophoresis. The serum albumin was kept in phosphate-buffered saline $(\mathrm{pH}$ 7.2) at concentrations of $15 \mathrm{mg}$ of protein $/ \mathrm{ml}$ and stored at $-20^{\circ} \mathrm{C}$. The albumin was radiolabeled with carrier-free ${ }^{125} \mathrm{I}$ by use of a glucose oxidase-lactoperoxidase procedure as described by the Bio-Rad Product Information Bulletin No. 1060 (Bio-Rad Laboratories, Richmond, CA).

${ }^{59} \mathrm{Fe}$-labeled $R B C$. An in vivo method of labeling $\mathrm{RBC}$ with ${ }^{59} \mathrm{Fe}$ was employed as described by Kopaniak et al. (24). Briefly, $300 \mu \mathrm{Ci}$ of $\left[{ }^{59} \mathrm{Fe}\right]$ ferrous citrate (New England Nuclear, Boston, MA) was incubated with $5 \mathrm{ml}$ of rabbit serum, and injected into the marginal ear vein of the rabbit to be used for in vivo labeling. The incorporation of ${ }^{59} \mathrm{Fe}$ into RBC was monitored, and as in the study by Kopaniak et al., the number of free or plasma-associated counts 48-72 $\mathrm{h}$ after injection of the radiolabel remained $<1 \%$ of the total counts in the blood with $99 \%$ localized in the RBC fraction. For experiments, $\sim 5$ $\mathrm{ml}$ of anticoagulated blood (10 $\mathrm{IU} / \mathrm{ml}$ of heparin) containing $\sim 6$ $\times 10^{6} \mathrm{cpm}$ of the ${ }^{59} \mathrm{Fe}-\mathrm{RBC}$ was obtained from the donor rabbit, and after counting, was infused into the recipient rabbit's circulation. The radioactivity and weight of $1 \mathrm{ml}$ of circulating blood was determined at the time of death of the animal under study.

Preparation of CVF. Cobra venom (Bioactive, Inc., Arlington, VA) was highly purified by DEAE-Sephadex chromatography as described by Cochrane and co-workers (25). The CVF was then treated with $p$ bromphenacyl bromide to remove any trace amounts of phospholipase $A_{2}$ (26). The biologic activity was hemolytically assayed (25) after which the CVF was stored at $-20^{\circ} \mathrm{C}$ until used. The intravenous dose of CFV employed in these studies was not associated with hemolysis of RBC.

Rabbit C5 assays. Hemolytic titers of rabbit C5 were determined by using a microtiter assay adopted from the procedure and reagent preparation described by Giclas and associates (23). Serum samples $(25 \mu \mathrm{l})$ were serially diluted twofold in glucose-gelatin veronal buffer (pH 7.35) after which $25 \mu \mathrm{l}$ of sheep erythrocytes $\left(5 \times 10^{7} / \mathrm{ml}\right)$ sensitized with antibody and incubated sequentially with complement components $\mathrm{C} 1, \mathrm{C} 4, \mathrm{C} 2$, and $\mathrm{C} 3$ was added to each well. Then, $25 \mu \mathrm{l}$ of normal rabbit serum treated with $2 \mathrm{M}$ potassium thiocyanate was added to each well. After incubation at $37^{\circ} \mathrm{C}$ for $60 \mathrm{~min}$, the plates were centrifuged and the hemolytic endpoints were determined. The endpoint titer was the reciprocal of the highest dilution at which hemolysis occurred. The results are expressed as the percent of initial hemolytic titer.

Bronchoalveolar lavage. At death, the lungs of the rabbits killed with intravenously administered sodium methohexital were carefully removed. After tying off one upper lung lobe for counting, a polypropylene catheter was gently inserted into the left lower lobe. After securing the catheter distal to the carina with silk suture, $15 \mathrm{ml}$ of sterile saline at $25^{\circ} \mathrm{C}$ was gently lavaged in and out of the lung a total of four times. This procedure routinely resulted in 10-12 ml of recovered volume. Recovered samples of $<9 \mathrm{ml}$ were not analyzed. The lavage samples were cooled to $3^{\circ} \mathrm{C}$ in wet ice after which the suspended cells were removed by centrifugation (300 $g$ for $10 \mathrm{~min}$ ). The radioactivity of the supernatant fluid was determined as described above, whereas the cell pellet was resuspended in phosphate-buffered saline for total leukocyte and differential count.

Histologic and electron microscopic examination of the lung. After the lavage as described above, the lavaged lobe was tied off, and the remainder of the lung prepared for histologic and electron microscopic examination as previously described (6). For this study, a Philips $\mathbf{4 0 0}$ $\mathrm{T}$ transmission electron microscope was used (Philips Electronic Instruments, Inc., Mahwah, NJ).

Neutrophil depletion. Rabbits were given $1.75 \mathrm{mg} / \mathrm{kg}$ intravenously of nitrogen mustard (Mustargen; Merck, Sharp \& Dohme; West Point, PA). Serial blood counts with differentials were obtained daily to document neutrophil depletion, and the animals were studied $72 \mathrm{~h}$ after nitrogen mustard administration. To protect neutrophil-depleted animals from infection, the rabbits received a combination of procaine penicillin $\mathbf{G}$ and dihydrostreptomycin sulfate (Combiotic; Pfizer Inc., New York, NY) at the time of nitrogen mustard administration and daily until studied. In addition, to prevent dehydration, rabbits received intravenous fluids 24 and $48 \mathrm{~h}$ after the initial nitrogen mustard infusion.

Blood cell counts. Counts of circulating leukocytes were performed in duplicate using a Coulter model ZBI counter (Coulter Electronics, Inc., Hialeah, FL). Differential counts were made on air-dried microscope slide smears stained with Wright's stain. Neutrophil counts were expressed as percent of the initial (baseline) value.

Sodium meclofenamate studies. Sodium meclofenamate monohydrate provided by Dr. Martin L. Black of Warner-Lambert Company (Ann Arbor, MI) was dissolved in sterile saline at a concentration of $1 \mathrm{mg} / \mathrm{ml}$. Rabbits given the drug received $10 \mathrm{mg} / \mathrm{kg}$ intravenously over a 30-min period before CVF administration, and an additional $3 \mathrm{mg} /$ $\mathrm{kg} \cdot \mathrm{h}$ by continuous infusion over the remainder of the experiment.

Statistical analysis. Data were analyzed with the $t$ test for paired or unpaired data. The $P$ value had to be $<0.05$ using a two-tailed test for the results to be considered statistically significant (27). In addition, the coefficient of correlation was computed for several variables with testing of the deviation of $r$ from 0 , or no correlation, by use of the $t$ test as outlined by Swinscow (28).

\section{Results}

Effect of CVF on circulating complement and neutrophil concentrations. Intravascular injection of $300 \mathrm{U}$ of CVF either alone or combined with hypoxia induced a decrease in hemolytic C5 concentrations, with presumed concomitant generation of $\mathrm{C} 5 \mathrm{a}$, to an average of $<10 \%$ of the initial value. As shown in a previous study (11), this degree of activity remained for the duration of the 4-h experiment. Exposure of the animals to $12 \%$ oxygen for a 10 -min period alone did not lead to a decrease in hemolytic C5 concentrations.

The effect of the CVF, with or without hypoxia, on circulating neutrophils was to cause a rapid neutropenia followed by a neutrophilia. As shown previously (11) the neutropenia occurred within $5 \mathrm{~min}$ of the bolus injection of CVF, led to virtual absence of neutrophils in the peripheral circulation, and was followed by a recovery of circulating neutrophils to levels above baseline by 2-4 h. No consistent changes in neutrophil number were seen in the animals subjected to hypoxia without CVF.

Effect of CVF and hypoxia on blood gases and pulmonary function. Fig. 1 shows the changes in arterial oxygen in rabbits treated with CVF alone, hypoxia alone, or CVF plus hypoxia. Before challenge, the mean $\mathrm{PaO}_{2}$ in all three groups was normal without significant differences between groups. As displayed in the figure, the mean decrease in $\mathrm{PaO}_{2}$ during the exposure to $12 \%$ oxygen was $\sim 40$ torr (mean $\mathrm{PaO}_{2} \pm \mathrm{SEM}=34.7 \pm 1.1$ ). Upon returning to breathing room air, the oxygen tension of the hypoxia alone group returned to baseline values, and throughout the remainder of the study, did not decrease significantly from baseline values. The group receiving CVF 


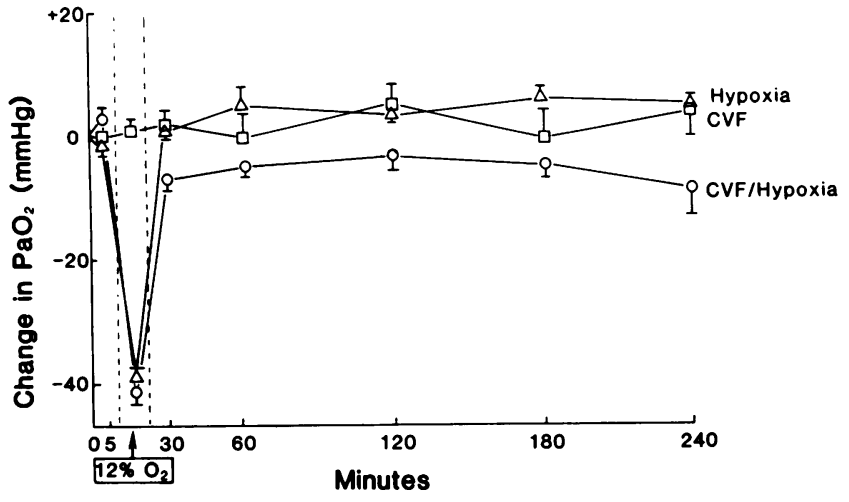

Figure 1. Change in $\mathrm{PaO}_{2}($ mean $\pm \mathrm{SEM})$ in rabbits treated with $\mathrm{CVF}$ alone at time 0 , hypoxia alone, or CVF followed by hypoxia. $n=5$, 6 , and 9 for each group, respectively. The latter group exhibited significant differences from the response in the groups treated with either stimulus alone.

alone also showed no significant changes from prechallenge values, or difference from the response to hypoxia. However, significant decreases in blood gas tensions were noted at 30 , 120 , and $240 \mathrm{~min}$ in the group subjected to both CVF and hypoxia when compared with the animals treated with CVF alone. At all time points from $30 \mathrm{~min}$ on, the response to hypoxia alone was significantly different from the response to CVF plus hypoxia. Whereas the magnitude of the drop in $\mathrm{PaO}_{2}$ in the latter group was not marked, the mean $\mathrm{PaO}_{2}$ never reached baseline values during the period of study.

Fig. 2 shows changes in $R_{\mathrm{L}}$ and $C_{\text {dyn }}$ for the three study groups. Again, baseline lung function was not significantly different between groups, but statistically significant differences in response to these stimuli were noted between the three groups. The animals exposed to both CVF and hypoxia had the most marked changes. Abnormalities were most pronounced at 120 min when a mean increase in the $R_{\mathrm{L}}$ to $145 \%$ of the

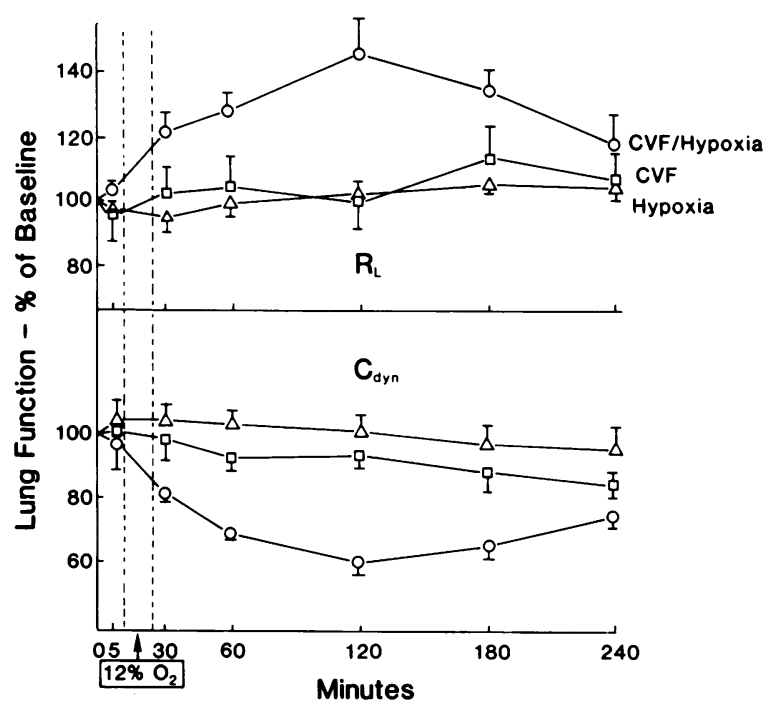

Figure 2. Changes in $R_{\mathrm{L}}$ and $C_{\mathrm{dyn}}$ in rabbits treated with CVF alone (time 0), hypoxia alone, or the combination of stimuli. Significant alterations occurred in the latter group that were maximal at 120 min. Neutrophil depletion prevented these changes in rabbits treated with $\mathrm{CVF} /$ hypoxia (data not shown). baseline value had occurred while $C_{\mathrm{dyn}}$ at this same time point had fallen to $59 \%$ of the baseline value $(P<0.01)$.

Effect of CVF with and without hypoxia on lung injury as assessed by lavage. As outlined in a previous study (11), the rabbits used for these experiments normally have $<1 \%$ neutrophils in the cells recovered by pulmonary lavage. As shown in Table I, the rabbits that undergo anesthesia, intubation, and the surgical procedures described above and then receive intravenous saline (control) at the start of the experiment had a slight increase in lavageable neutrophils after $4 \mathrm{~h}$ of study $(1.3 \pm 0.6 \%$, mean \pm SEM). If $300 \mathrm{U}$ of CVF was given intravenously to rabbits, the percentage increased to a mean of $7.8 \%$, but as noted below, this was not accompanied by an increase in lavageable albumin. When the animals were subjected to $10 \mathrm{~min}$ of $12 \%$ oxygen alone, and were killed and lavaged $4 \mathrm{~h}$ later, the percent neutrophils increased to $4.2 \pm 1.3 \%(P<0.05$ vs. saline control). When the $300 \mathrm{U}$ of CVF was followed 10 min after the infusion by $10 \mathrm{~min}$ of $12 \%$ oxygen, the percent neutrophils in the 14 animals studied increased to $11.2 \% \pm 1.9 \%$ $(P<0.01$ vs. saline control).

Permeability in the lungs of the animals to radiolabeled albumin was determined by assessing the radioactivity present in the lavage both 1 and $4 \mathrm{~h}$ after challenge. The radiolabel was injected $30 \mathrm{~min}$ before the animal was killed so that the measured permeability to albumin across the alveolar-capillary membrane was limited to that $30-\mathrm{min}$ period alone. In this way, the period under study was not one in which hemodynamic changes were occurring that could influence the interpretation of the data (see below). As shown in Fig. 3, after CVF or hypoxia alone, the mean permeability as expressed by the lavage/plasma ratio for ${ }^{125}$ I-RSA was not significantly elevated when compared with the control experiments where saline was given. In addition, combining the CVF and hypoxia did not increase lavageable albumin at an early time point $(1 \mathrm{~h})$. On the other hand, $4 \mathrm{~h}$ after the combination of CVF plus hypoxia, increased values for permeability over a wide range were noted ( $P<0.01$ for all groups compared with $\mathrm{CVF} /$ hypoxia group).

Effect of neutrophil depletion on pulmonary changes produced by CVF and hypoxia. On the day that the animals treated $72 \mathrm{~h}$ earlier with nitrogen mustard were studied, two of the four animals had no neutrophils observed on peripheral blood counts and the two remaining animals had $<500$ neutrophils $/ \mathrm{mm}^{3}$. Normally, the mean neutrophil count is $\sim 2,500 / \mathrm{mm}^{3}$. The mean hematocrit was $40 \%$ with the mean lymphocyte and platelet counts being $1,200 / \mathrm{mm}^{3}$ and $273,000 /$

Table I. Total Leukocytes and Percent Neutrophils in Lavages

\begin{tabular}{lrlr}
\hline Group & \multicolumn{1}{c}{$n$} & Total leukocytes* & Neutrophils \\
\hline & & $\times 10^{\circ}$ & \multicolumn{1}{l}{$\%$} \\
Saline & 8 & $5.8 \pm 1.3$ & $1.3 \pm 0.6$ \\
CVF & 9 & $7.9 \pm 1.5$ & $7.8 \pm 4.3$ \\
Hypoxia & 5 & $5.8 \pm 1.2$ & $4.2 \pm 1.3$ \\
CVF/hypoxia & 14 & $7.2 \pm 1.3$ & $11.2 \pm 2.4$ \\
CVF/hypoxia (-PMN) & 4 & $3.4 \pm 0.6$ & $1.1 \pm 0.6$ \\
m-CVF/hypoxia & 3 & $4.5 \pm 0.4$ & $1.7 \pm 0.3$
\end{tabular}

Abbreviations: CVF, cobra venom factor; $-\mathrm{PMN}$, polymorphonuclear neutrophil depletion; m, sodium meclofenamate.

* Values are mean \pm SEM. 


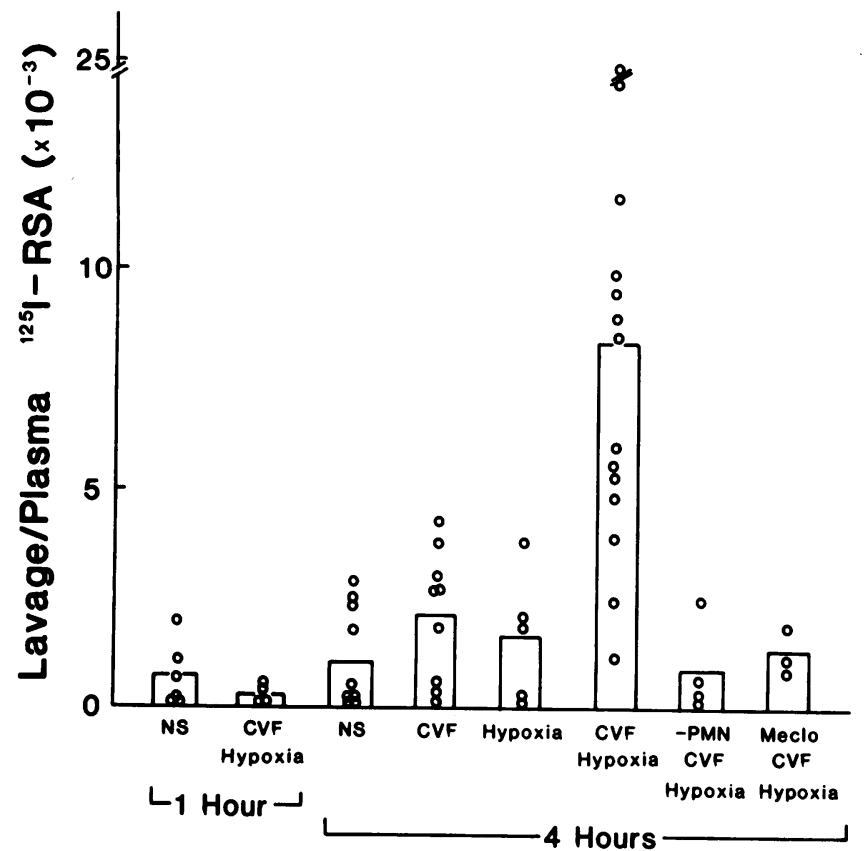

Figure 3. Radiolabeled albumin in lavage expressed as a ratio to plasma albumin (counts per minute per milliliter of lavage/counts per minute per milliliter of plasma) in animals treated with normal saline (NS) alone (controls), CVF alone, hypoxia alone, or the combination of CVF/hypoxia. The radiolabel was injected $30 \mathrm{~min}$ before sacrifice of the animals whereas the CVF and hypoxia were given at the beginning of the experiments. Only the combination of CVF/ hypoxia led to significant increases in lavage albumin $4 \mathrm{~h}$ after challenge ( $P<0.01$ for $C V F /$ hypoxia compared with all groups). Neutrophil depletion (-PMN) and meclofenamate treatment (Meclo) blocked the increased albumin accumulation seen with CVF/hypoxia.

$\mathrm{mm}^{3}$, respectively. These values are normal for our laboratory except for the lymphocyte count, which averages $\sim 4,500$ / $\mathrm{mm}^{3} .4 \mathrm{~h}$ after challenge with CVF and hypoxia, the mean percentage of neutrophils in the lavages was $1.1 \%$ (Table I). The mean accumulation of albumin in the lavage was not elevated (Fig. 3). No consistent changes in arterial oxygen occurred during the 4-h experiment, and mean $\mathrm{PaO}_{2}$ at $4 \mathrm{~h}$ was not decreased from baseline values. In addition, the mean $R_{\mathrm{L}}$ for this experimental group was never $>110.2 \%$ of the baseline value during the study while $C_{\text {dyn }}$ did not fall below $88 \%$. Thus, neutrophil depletion prevented the increase in lavage neutrophils and albumin associated with CVF/hypoxia challenge as well as the physiologic alterations (increased $R_{\mathrm{L}}$, decreased $C_{\text {dyn }}$ and $\mathrm{PaO}_{2}$ ) noted when neutrophils were present.

Effect of CVF plus hypoxia on lung albumin and blood. When both ${ }^{125} \mathrm{I}-\mathrm{RSA}$ and ${ }^{59} \mathrm{Fe}-\mathrm{RBC}$ were infused into animals, more extensive assessments of lung fluid, albumin, and blood could be performed. In particular, the wet/dry weights of bloodless lung, the lung extravascular water, the lung/plasma ratio of ${ }^{125}$ I-RSA (counts per minute per gram of dry bloodless lung/counts per minute per milliliter of plasma), and lung blood (gram of blood/gram of dry bloodless lung) were determined. While the mean values of both wet/dry weights of lung and extravascular water were greater in the CVF/hypoxiatreated rabbits than in the other groups, the differences were not statistically significant. In addition, the accumulation of albumin in the lavages did not correlate with either wet/dry weights or lung extravascular water. On the other hand, lavage

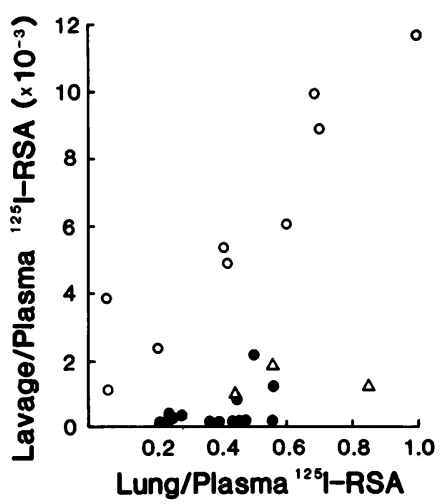

Figure 4. Lavage/plasma ${ }^{125} \mathrm{I}$ RSA (counts per minute per milliliter of lavage/counts per minute per milliliter of plasma) vs. lung/plasma ${ }^{125} \mathrm{I}$ RSA (counts per minute per gram of dry bloodless lung/ counts per minute per milliliter of plasma) for three groups of rabbits: rabbits receiving CVF/hypoxia $4 \mathrm{~h}$ before sacrifice (0), rabbits receiving saline alone one or 4 $\mathrm{h}$ before sacrifice or CVF/hypoxia $1 \mathrm{~h}$ before sacrifice $(\bullet)$,

and rabbits receiving meclofenamate plus CVF/hypoxia $4 \mathrm{~h}$ before sacrifice $(\Delta)$. In the first group, the relationship between these variables is significant ( $r=0.94, P<0.001)$, and as the bloodless lung radioactivity increases, so does the lavage albumin. In saline controls or rabbits challenged $1 \mathrm{~h}$ before sacrifice, this relationship was not apparent. Meclofenamate treatment prevented an increase in lavage albumin without decreasing lung-associated albumin, implying there may still be interstitial accumulation in this circumstance without transit into the alveolus.

albumin did correlate with the increased lung/plasma ratio of ${ }^{125}$ I-RSA noted in rabbits receiving both CVF and hypoxia 4 h before death $(r=0.94, P<0.001$; Fig. 4). In addition, as shown in Fig. 5, lung blood was noted to be significantly correlated with the radiolabeled albumin in the lavage for the control (saline injected) and experimental groups (CVF/hypoxia 1 and $4 \mathrm{~h}$ before sacrifice).

Effect of sodium meclofenamate treatment on lung injury produced by CVF plus hypoxia. One reason for selecting hypoxia as the second stimulus for challenge was the observation that hypoxia leads to generation of prostaglandins (14). Therefore, in three experiments, meclofenamate was given before and after CVF/hypoxia as described above. This altered the response to $\mathrm{CVF} /$ hypoxia in several ways. First, $\mathrm{PaO}_{2}$ did not fall significantly from baseline values (data not shown), Other indices of lung function were not measured during these experiments because of simultaneous determinations of he-

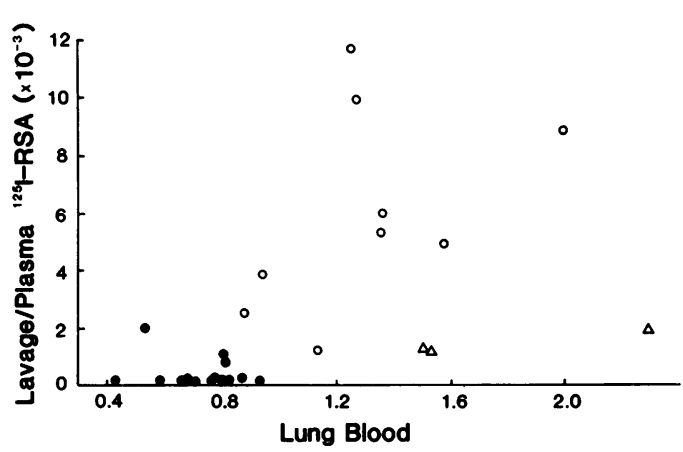

Figure 5. Lung blood (gram of blood/gram of dry bloodless lung) is plotted vs. the lavage albumin (counts per minute per milliliter of lavage/counts per minute per milliliter of plasma) for the same groups listed in Fig. 4. For the groups not receiving meclofenamate, a significant correlation exists $(r=0.76, P<0.01)$. Meclofenamate treatment reduced albumin in the lavage without decreasing lung blood. 
modynamic alterations (see below). The meclofenamate infusion did prevent increases in the lavage/plasma ratio of ${ }^{125} \mathrm{I}$ RSA (Fig. 3), and this was associated with a significant decrease in lavage neutrophils $(P<0.01, \mathrm{CVF} /$ hypoxia vs. meclofenamate-CVF/hypoxia, Table I). Meclofenamate did not, however, prevent the precipitous fall in circulating neutrophils after CVF. As shown in Fig. 4, when meclofenamate was given with CVF/hypoxia, the lung/plasma ratio of ${ }^{125} \mathrm{I}-\mathrm{RSA}$ could still be increased (compared with control animals) without an associated increase in the lavage/plasma ratio for albumin. Fig. 5 also shows that meclofenamate did not prevent the increase in lung blood found when CVF/hypoxia was given, but did prevent increases in lavage albumin.

Hemodynamic measurements. In six experiments where rabbits received CVF and hypoxia, pulmonary artery pressure (PAP) and cardiac output were monitored. In three experiments, the rabbits were also given meclofenamate whereas in the other three, only CVF/hypoxia was given. With CVF/hypoxia, the mean PAP was elevated from baseline values immediately after the bolus of CVF was given and again during the exposure to hypoxia (Fig. 6). Subsequently, the mean PAP returned to baseline values and did not increase again during the remainder of the experiment. In the experiments where meclofenamate was given before and for $4 \mathrm{~h}$ after CVF/hypoxia, the mean PAP did not increase after CVF infusion. The increases in PAP associated with exposure to hypoxia were not blocked, however. Pressures measured subsequently were again not significantly different from baseline values in this latter group.

In the group of animals that were treated with CVF/ hypoxia, the cardiac index fell from a baseline value of $0.30 \pm 0.01 \mathrm{liter} / \mathrm{min} \cdot \mathrm{kg}($ mean $\pm \mathrm{SEM})$ to a low value of $0.19 \pm 0.03 \mathrm{liter} / \mathrm{min} \cdot \mathrm{kg}$ at $5 \mathrm{~min}$ after CVF. During hypoxia, this value rose to above baseline values $(0.35 \pm 0.06$ liter/ $\mathrm{min} \cdot \mathrm{kg}$ ), and remained above baseline values for the remainder of the experiment. With meclofenamate pretreatment, cardiac index did not fall from baseline values $(0.23 \pm 0.05$ liter/ $\mathrm{min} \cdot \mathrm{kg}$ ) after CVF infusion. As in the other group, the cardiac index increased during hypoxia to above baseline values, and remained above baseline the remainder of the experiment.

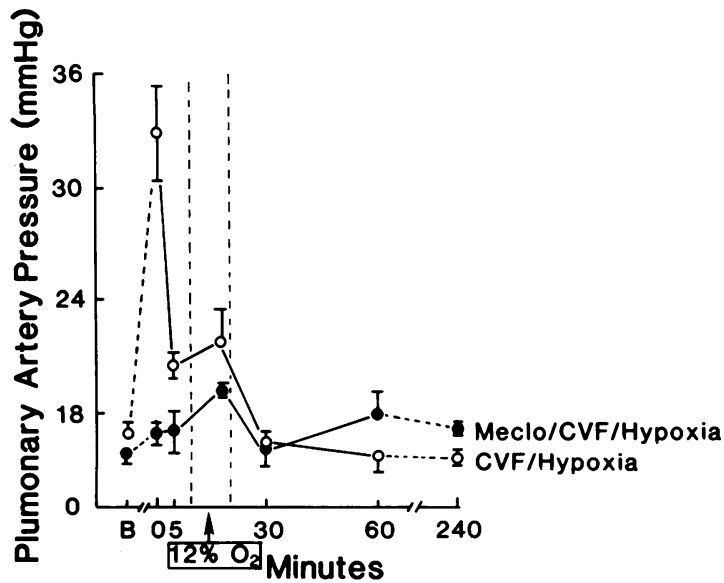

Figure 6 . The mean pulmonary artery pressure $( \pm$ SEM) for animals treated with $\mathrm{CVF} /$ hypoxia, or meclofenamate followed by CVF and hypoxia. Meclofenamate treatment prevents the acute rise in PAP without blunting the increase in PAP caused by hypoxia. These hemodynamic events are short, and have returned to baseline values by the 30 -min time point. $n=3$ for both groups.
Effect of CVF with and without hypoxia on lung structure. The intravenous administration of CVF to rabbits caused neutrophil accumulation in the capillaries of the lung that was apparent on histologic examination of the lung $4 \mathrm{~h}$ after challenge. This histologic picture was indistinguishable from that seen after intravenous rabbit $\mathrm{C} 5 \mathrm{a}$ as shown in a previous study (11). Challenge with hypoxia alone did not cause any apparent abnormalities in light or electron microscopy during the same time period. With the combination of insults, the capillaries again contained many neutrophils, but in addition, the alveolar air spaces occasionally contained neutrophils. These differences between the histology of animals treated with CVF alone or CVF plus hypoxia were not striking on light microscopy. Examination by electron microscopy also revealed mild abnormalities. As shown in Fig. 7, animals receiving CVF and hypoxia had distended vessels full of neutrophils and red blood cells (Fig. $7 a$ ) with higher magnifications (Fig. 7 b) showing vesicles within endothelial cells. These findings were not as prominent in animals treated with either insult alone. No evidence of damage to alveolar epithelial-lining cells was noted in any group of animals. In addition, no evidence of intraalveolar or interstitial hemorrhage was found.

\section{Discussion}

This study demonstrates that complement activation with $\mathrm{CVF}$, as an isolated challenge, does not lead to an increase in
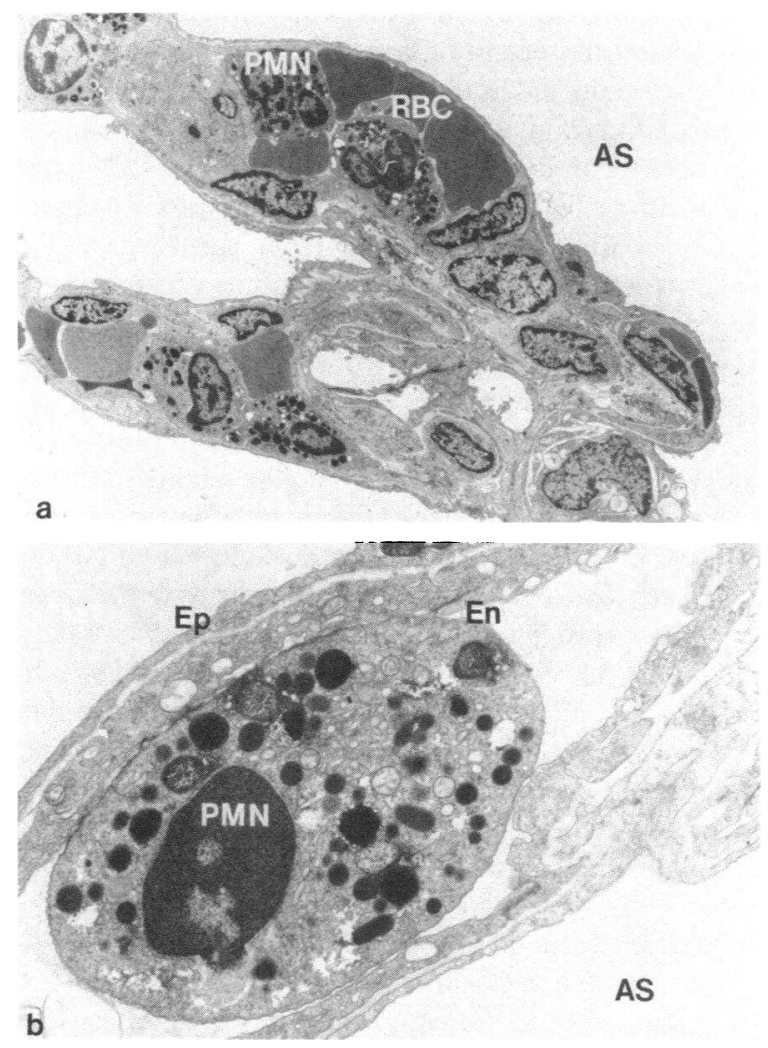

Figure 7. Transmission electron micrographs of two animals treated with CVF and hypoxia $4 \mathrm{~h}$ before death. (a) Neutrophils (PMN) and erythrocytes (RBC) are prominent in dilated vessels. The air spaces (AS) in the lung are not filled with fluid (original magnification: $\times 1,838$ ). (b) A neutrophil is noted to be closely approximated to the endothelial cell (En). Vesicles are prominent within the endothelial cells. The epithelial cell $(\mathrm{Ep})$ is normal in appearance (original magnification: $\times 7,838$ ). 
lavage radiolabeled albumin or appreciably alter lung function as measured by $\mathrm{PaO}_{2}$ and tests of lung mechanics $\left(R_{\mathrm{L}}\right.$ and $\left.C_{\text {dyn }}\right)$ in this model. These observations are consistent with our earlier reported findings (11), and further define the lack of physiologic effect of complement activation alone on the lung in the rabbit. In addition, however, we have shown that by combining complement activation with another challenge (hypoxia) that alone will not alter permeability or lung function, we can measureably alter lavage albumin, $\mathrm{PaO}_{2}, R_{\mathrm{L}}$, and $C_{\mathrm{dyn}}$.

Recent reports have raised the possibility that complement activation with generation of $\mathrm{C5}$-derived fragments will cause neutrophil deposition in the pulmonary vasculature and subsequent lung injury (29). Whereas there is no question from experimental evidence that generation of complement-derived chemotactic fragments will cause sequestration of neutrophils within the lungs $(1,10,11,30)$, it is possible that other stimuli may be needed in addition to complement activation for significant pulmonary alterations to occur $(11,13,31)$. For example, we recently noted that complement activation together with prostaglandin administration increased lung permeability to albumin in a rabbit model (13). Based on these observations, it is possible to speculate that complement activation may play a part in acute lung injury by placing neutrophils in the target organ (lung), but other stimuli are needed to enhance their injurious potential.

For the present study, hypoxia was chosen as the second stimulus for two reasons. First, in shock lung syndrome, a disease where complement activation with pulmonary neutrophil sequestration has been implicated in its pathogenesis (2), hypoxia and/or poor tissue oxygenation are frequently found $(32,33)$. Second, during hypoxia, prostaglandins are generated (14), and as shown by Wedmore and Williams (12) plus Issekutz (34) in rabbit skin, and by our laboratory in rabbit lung (13), the combination of a chemotactic agent with prostaglandins can lead to an increase in albumin accumulation in the target tissue. The timing of the hypoxic challenge was designed to occur at a time known from previous observations that the pulmonary vasculature contains many neutrophils (35). The challenge was limited to $10 \mathrm{~min}$ because we found that although anesthetized rabbits could be maintained on $12 \%$ oxygen for up to $4 \mathrm{~h}$ with stable lung function and systemic blood pressure, the CVF stimulus followed by persistent $12 \%$ oxygen led to death of some animals over the time course of the experiments (Larsen, G. L., unpublished data). The periods of time chosen to assess permeability $(0.5-1 \mathrm{~h}$ and 3.5-4 $\mathrm{h}$ after CVF) were deliberately separated from the challenges to avoid interference by hemodynamic events due to the stimuli that could have altered albumin accumulation in the lung due to pressure changes alone. As shown in Fig. 6 , the PAP was unchanged from baseline values during the time periods albumin accumulation was being measured.

The albumin accumulation in the lung lavage of animals treated with CVF and hypoxia, although statistically different from the other groups, varied greatly in magnitude. The differences in this value between experiments in this group were not readily apparent upon examining the data. The animals were not acidotic during the last $3.5 \mathrm{~h}$ of the experiment, and thus bradykinin production because of acidosis could not be implicated as a cause of the increased protein accumulation (36). No obvious differences in neutrophil counts before or after challenge were noted among the animals, and the $\mathrm{PaO}_{2}$ upon exposure to $12 \%$ oxygen did not correlate with the albumin accumulation in the lavages. Despite this variability in albumin accumulation, this group was clearly different from the two other groups challenged with hypoxia alone or CVF alone in regard to lavage findings, $\mathrm{PaO}_{2}$, and lung mechanics. It is possible that slight alterations in the timing of the two events (CVF and hypoxia) could markedly increase or decrease these findings. This remains to be explored.

The lack of effect of intravenous CVF alone found in this study and a previous study (11) is in contrast to the results of Till et al. (10) and Ward and co-workers (37). Several potential differences in the studies may explain these discrepancies. First, their experimental animal is the rat, and as reported by Martin and associates (38), rat lung explants are more sensitive to oxidant injury than rabbit lung explants. Because oxygen radical production appears to be important in CVF-induced pulmonary changes (37), the relative resistance of the lung of the rabbit to this injury may partially explain the differences in the studies. In addition, however, the definition of pulmonary alterations that signify injury is different. In the studies from Michigan $(10,37)$, altered permeability is defined through use of radiolabeled albumin or IgG injected intravenously. At sacrifice, the pulmonary vasculature was perfused free of blood, and the radioactivity remaining in the lung was determined and related to radioactivity in the blood. This measure of vascular permeability does not address whether the label is in the pulmonary interstitium (i.e., crossed the pulmonary endothelium) or in the alveolus (crossed both the pulmonary endothelium and epithelium). On the other hand, the primary marker of altered permeability to albumin used in this and our previous study (11) was lavage albumin. Thus, the radiolabeled protein had to traverse both barriers to be measured, making our assessment more stringent. In this study, delineation of the accumulation of interstitial protein was attempted by use of the two radiolabels ( ${ }^{125} \mathrm{I}-\mathrm{RSA}$ and $\left.{ }^{59} \mathrm{Fe}-\mathrm{RBC}\right)$. Thus, the lung/plasma ${ }^{125} \mathrm{I}-\mathrm{RSA}$ represents both interstitial and lavage albumin whereas the lavage/plasma ${ }^{125} \mathrm{I}$-RSA represents only lavageable albumin. As shown in Fig. 4, in animals receiving CVF/hypoxia $4 \mathrm{~h}$ before death, a high correlation existed between the two measurements. It is important to note, however, that a high lung/plasma ${ }^{125}$ I-RSA was not always associated with a high lavage/plasma ${ }^{125}$ I-RSA. For example, the highest lung/plasma ${ }^{125} \mathrm{I}-\mathrm{RSA}$ was in a meclofenamatetreated rabbit that received $\mathrm{CVF} /$ hypoxia, and yet lavage albumin was normal. For the meclofenamate group as a whole, the mean lung/plasma ${ }^{125} \mathrm{I}-\mathrm{RSA}$ was greater than in controls, and yet lavage albumin was normal. This might imply that an arachidonic acid product of the cyclooxygenase pathway is necessary to allow albumin to cross this epithelial barrier. In addition, because meclofenamate will not block leukotriene production, it is possible that lipoxygenase products of arachidonic acid metabolism help mediate the interstitial accumulation of protein. These possibilities require more study.

The abolition of the albumin accumulation in animals made neutropenic before challenge implies that neutrophils are important in the development of this altered permeability. This finding is similar to the results recently reported by Heflin and Brigham (39) in chronically instrumented sheep, where neutrophil depletion prevented the increased lung vascular permeability to fluid and protein following endotoxin administration. Similar findings have been found in animal models of pulmonary edema associated with air emboli in sheep (40) and glass bead pulmonary embolization in dogs (41). Thus, in 
this as in other models, the neutrophil may play an important role in altering vascular permeability in the lung. However, in our study as well as the ones cited above, it must be recognized that nitrogen mustard used as a neutrophil-depleting agent might have other effects on the lung. Neutrophil repletion experiments would make the argument stronger, but are presently technically difficult, and have the added drawback that infused cells are not functionally equivalent to normal neutrophils in vivo. These problems are being addressed in ongoing experiments so that improved techniques for reconstituting functional neutrophils will be available. In defense of the depletion studies, however, it is important to note that nitrogen mustard acts very rapidly on dividing cells, and was given $3 \mathrm{~d}$ before the experiments were conducted. The fact that the other hematologic parameters measured after this treatment were normal or near normal suggests the effects seen may be due to neutrophil depletion alone.

The effect of meclofenamate in various animal models of lung injury has differed. For example, in the sheep model of endotoxin-induced lung injury, Snapper and co-workers (42) found meclofenamate attenuated the early pulmonary hypertension after endotoxin infusion, but had no effect on the leukopenia induced by this insult. These results are similar to our finding. These authors note, however, that cyclooxygenase blocking agents do not significantly attenuate the late phase of increased permeability of the pulmonary vascular endothelium. Again, differences in animal models and definition of injury may explain what appear to be divergent results. In another sheep model of injury where the insult is repeated bolus injections of autologous zymosan activated plasma, Petrowski and associates (43) found meclofenamate inhibited hypoxemia but not the pulmonary hypertensive response or increased lymph flow and protein clearance. These results regarding the effect of meclofenamate on protein fluxes again are different, but may relate to our definition of permeability as an increase in lavage albumin although their measure is through assessment of protein in lymph. The fact that meclofenamate significantly decreased lavage albumin in our model while interstitial albumin remained high (lung/plasma ${ }^{125}$ I-RSA) would support that argument.

The mechanism through which meclofenamate (and the subsequent cyclooxygenase inhibition) work to decrease accumulation of albumin in the lavage is unknown. Because vasodilator prostaglandins such as prostaglandin $\mathrm{I}_{2}$ are produced during hypoxia, and could lead to a recruitment of pulmonary capillaries and/or an increase in capillary surface area, and this in itself might account for some of the increase in lavage albumin, the relative volume of blood in the lung was evaluated. As noted in Fig. 5, there was a correlation between increases in lung blood and increases in lavage albumin. However, meclofenamate prevented increases in the latter variable while lung blood remained high, thus implying that cyclooxygenase products did not have their effect through an increase in capillary volume or surface area. It is interesting to note that meclofenamate treatment did prevent increases in neutrophils in the lavage of CVF/hypoxia-treated animals. Thus, its mechanism of action may relate to preventing neutrophil migration across the epithelial barrier. It is important to note that Issekutz (34) has reported vasodilating inflammatory mediators such as prostaglandins $E_{1}$ and $E_{2}$ enhanced neutrophil infiltration in rabbit skin in response to chemotactic stimuli. If a similar effect of chemotactic factors and prostaglandins occurs in the lung, then cyclooxygenase inhibition could decrease neutrophil emigration. This remains speculative at this point.

The pathophysiology of the changes in $R_{\mathrm{L}}$ and $C_{\mathrm{dyn}}$ remain to be explained. It is possible that these mild alterations are related to fluid and protein accumulation in the pulmonary interstitium and alveoli. The abrogation of these changes with neutrophil depletion and subsequent prevention of increased albumin flux would suggest this. However, it also is possible that mediators generated by the combined CVF/hypoxia stimuli might also mediate some or all of these changes. These possibilities remain to be explored.

This study has demonstrated complement activation with CVF in rabbits, as an isolated event, does not lead to measureable changes in lung function over a 4-h period, and does not lead to increased albumin accumulation across the lung at either an early or a late time point. The study does show, however, that by combining complement activation with a common physiologic event (hypoxia) that in itself does not harm the lung, we can induce measurable changes in lung structure and function.

\section{Acknowledgments}

The authors express their appreciation to Brenda Mitchell, Alan Goins, and Mary Wilcox for their technical assistance, Jan Henson for her assitance with the transmission electron microscopy, and Georgia Wheeler for her preparation of the manuscript.

This study was supported by grants HL-27063, HL-21565, and HL-31376 from the National Institutes of Health.

\section{References}

1. Craddock, P. R., J. Fehr, K. L. Brigham, R. S. Kronenberg, and H. S. Jacob. 1977. Complement and leukocyte-mediated pulmonary dysfunction in hemodialysis. N. Engl. J. Med. 296:769-774.

2. Hammerschmidt, D. E., L. J. Weaver, L. D. Hudson, P. R. Craddock, and H. S. Jacob. 1980. Association of complement activation and elevated plasma-C5a with adult respiratory distress syndrome. Lancet. I:947-949.

3. Chenoweth, D. E., J. G. Rowe, and T. E. Hugli. 1979. A modified method for chemotaxis under agarose. J. Immunol. Methods. 25:337-353.

4. Webster, R. O., S. R. Hong, R. B. Johnston, and P. M. Henson. 1980. Biological effects of the human complement fragments $\mathrm{C} 5 \mathrm{a}$ and C5a des Arg on neutrophil function. Immunopharmacology. 2:201219.

5. Sacks, T., C. F. Moldow, P. R. Craddock, T. K. Bowers, and H. S. Jacob. 1978. Oxygen radicals mediate endothelial cell damage by complement-stimulated granulocytes. J. Clin. Invest. 61:1161-1167.

6. Larsen, G. L., K. McCarthy, R. O. Webster, J. Henson, and P. M. Henson. 1980. A differential effect of C5a and C5a des Arg in the induction of pulmonary inflammation. Am. J. Pathol. 100:179192.

7. Desai, U., D. L. Kreutzer, H. Showell, C. V. Arroyave, and P. A. Ward. 1979. Acute inflammatory pulmonary reactions induced by chemotactic factors. Am. J. Pathol. 96:71-84.

8. Shaw, J. O., P. M. Henson, J. Henson, and R. O. Webster. 1980. Lung inflammation induced by complement-derived chemotactic fragments in the alveolus. Lab. Invest. 42:547-558.

9. Stimler, N. P., T. E. Hugli, and C. M. Bloor. 1980. Pulmonary injury induced by $\mathrm{C} 3 \mathrm{a}$ and $\mathrm{C} 5 \mathrm{a}$ anaphylatoxins. Am. J. Pathol. 100: 327-348.

10. Till, G. O., K. J. Johnson, R. Kunkel, and P. A. Ward. 1982. Intravascular activation of complement and acute lung injury. Dependency on neutrophils and toxic oxygen metabolites. J. Clin. Invest. 69: 1126-1135. 
11. Webster, R. O., G. L. Larsen, B. C. Mitchell, A. J. Goins, and P. M. Henson. 1982. Absence of inflammatory lung injury in rabbits challenged intravascularly with complement-derived chemotactic factors. Am. Rev. Respir. Dis. 125:335-340.

12. Wedmore, C. V., and T. J. Williams. 1981. Control of vascular permeability by polymorphonuclear leukocytes in inflammation. Nature (Lond.). 289:646-650.

13. Henson, P. M., G. L. Larsen, R. O. Webster, B. C. Mitchell, A. J. Goins, and J. E. Henson. 1982. Pulmonary microvascular alterations and injury induced by complement fragments: synergistic effect of complement activation, neutrophil sequestration, and prostaglandins. Ann. NY Acad. Sci. 384:287-300.

14. Said, S. I., T. Yoshida, S. Kitamura, and C. Vreim. 1974. Pulmonary alveolar hypoxia: release of prostaglandins and other humoral mediators. Science (Wash. DC). 185:1181-1183.

15. Mead, J., and C. Collier. 1959. Relation of volume history of lungs to respiratory mechanics in anesthetized dogs. J. Appl. Physiol. 14:669-678.

16. Caldwell, E. J., and D. L. Fry. 1969. Pulmonary mechanics in the rabbit. J. Appl. Physiol. 27:280-285.

17. Von Neergard, K., and K. Wirz. 1927. A method of measuring lung elasticity in living humans, particularly in the presence of emphysema. Z. Klin. Med. 105:35-50.

18. Mead, J., and J. L. Whittenberger. 1953. Physical properties of human lung measured during spontaneous respiration. J. Appl. Physiol. 5:779-796.

19. Drazen, J. M., S. H. Loring, and R. Regan. 1976. Validation of an automated determination of pulmonary resistance by electrical subtraction. J. Appl. Physiol. 40:110-113.

20. Owen-Thomas, J. B., and J. T. Reeves. 1969. Hypoxia and pulmonary arterial pressure in the rabbit. J. Physiol. (Lond.). 201:665672.

21. Reybrouck, T., A. Amery, L. Billiet, R. Fagard, and H. Stijns. 1978. Comparison of cardiac output determined by a carbon dioxiderebreathing and direct Fick method at rest and during exercise. Clin. Sci. Mol. Med. 55:445-452.

22. Pearce, M. L., J. Yamashita, and J. Beazell. 1965. Measurement of pulmonary edema. Circ. Res. 16:482-488.

23. Giclas, P. C., P. J. Keeling, and P. M. Henson. 1981. Isolation and characterization of the third and fifth components of rabbit complement. Mol. Immunol. 18:113-123.

24. Kopaniak, M. M., A. C. Issekutz, C. E. Burrowes, and H. Z. Movat. 1980. The quantitation of hemorrhage in the skin. Measurement of hemorrhage in the microcirculation in inflammatory lesions and related phenomena. Proc. Soc. Exp. Biol. Med. 163:126-131.

25. Cochrane, C. G., H. J. Muller-Eberhard, and B. B. Aiken. 1970. Depletion of plasma complement in vivo by a protein of cobra venom: its effect on various immunologic reactions. J. Immunol. 105: 55-69.

26. Shaw, J. O., M. F. Roberts, R. J. Ulevitch, P. M. Henson, and E. A. Dennis. 1978. Phospholipase $A_{2}$ contamination of cobra venom factor preparations. Biological role in complement-dependent in vivo reactions and inactivation with p-bromphenacyl bromide. Am. J. Pathol. 91:517-528.
27. Remington, R. D., and M. A. Schork. 1970. Statistics with application to the biological and health sciences. Englewood Cliffs: Prentice Hall. 418 pp.

28. Swinscow, T. D. V. 1976. Statistics at square one. XIX. Correlation. Br. Med. J. 2:747-748.

29. Jacob, H. S., P. R. Craddock, D. E. Hammerschmidt, and C. F. Moldow. 1980. Complement-induced granulocyte aggregation. An unsuspected mechanism of disease. N. Engl. J. Med. 302:789-794.

30. Hohn, D. C., A. J. Meyers, S. T. Gherini, A. Beckman, R. E. Markison, and A. M. Churg. 1980. Production of acute pulmonary injury by leukocytes and activated complement. Surgery. 88:48-58.

31. Cooper, J. D., J. W. D. McDonald, M. Ali, E. Menkes, J. Masterson, and P. Klement. 1980. Prostaglandin production associated with the pulmonary vascular response to complement activation. Surgery (St. Louis). 88:215-222.

32. Hyers, T. M. 1981. Pathogenesis of adult respiratory distress syndrome: current concepts. Semin. Respir. Med. 2:104-108.

33. Rinaldo, J. E., and R. M. Rogers. 1982. Adult respiratory distress syndrome. Changing concepts of lung injury and repair. $N$. Engl. J. Med. 306:900-909.

34. Issekutz, A. C. 1981. Effect of vasoactive agents on polymorphonuclear leukocyte emigration in vivo. Lab. Invest. 45:234-240.

35. Henson, P. M., K. McCarthy, G. L. Larsen, R. O. Webster, P. C. Giclas, R. B. Dreisen, T. E. King, and J. O. Shaw. 1979. Complement fragments, alveolar macrophages, and alveolitis. Am. J. Pathol. 97:93-110.

36. O'Brodovich, H. M., S. A. Stalcup, L. M. Pang, J. S. Lipset, and R. B. Mellins. 1981. Bradykinin production and increased pulmonary endothelial permeability during acute respiratory failure in unanesthetized sheep. J. Clin. Invest. 67:514-522.

37. Ward, P. A., G. O. Till, R. Kunkel, and C. Beauchamp. 1983. Evidence for role of hydroxyl radical in complement and neutrophildependent tissue injury. J. Clin. Invest. 72:789-801.

38. Martin, W. J., J. E. Gadek, G. W. Hunninghake, and R. G. Crystal. 1981. Oxidant injury of lung parenchymal cells. J. Clin. Invest. 68:1277-1288.

39. Heflin, A. C., and K. L. Brigham. 1981. Prevention by granulocyte depletion of increased vascular permeability of sheep lung following endotoxemia. J. Clin. Invest. 68:1253-1260.

40. Flick, M. R., A. Perel, and N. Staub. 1981. Leukocytes are required for increased lung microvascular permeability after microembolism in sheep. Circ. Res. 48:344-351.

41. Johnson, A., and A. Malik. 1980. Effect of granulocytopenia on extravascular lung water content after microembolization. Am. Rev. Respir. Dis. 122:561-566.

42. Snapper, J. R., G. R. Bernard, J. M. Hinson, A. A. Hutchison, J. E. Loyd, M. L. Ogletree, and K. L. Brigham. 1983. Endotoxemiainduced leukopenia in sheep. Correlation with lung vascular permeability and hypoxemia but not with pulmonary hypertension. Am. Rev. Respir. Dis. 127:306-309.

43. Perkowski, S. Z., A. M. Havill, J. T. Flynn, and M. H. Gee. 1983. Role of intrapulmonary release of eicosanoids and superoxide anion as mediators of pulmonary dysfunction and endothelial injury in sheep with intermittent complement activation. Circ. Res. 53:574583. 